\title{
A MULTI HAZARD PERSPECTIVE IN FLOOD AND DROUGHT VULNERABILITY: CASE STUDY OF MALAWI
}

\author{
Tamara F. KAMANGA ${ }^{1,4}$ (D), Sarintip TANTANEE ${ }^{2}$ (D), \\ Faidess D. MWALE ${ }^{3}$ iD, PAnu BURANAJARUKORN ${ }^{4}$
}

DOI: 10.21163/GT_2020.151.12

\begin{abstract}
:
The prominence of vulnerability assessments cannot be over emphasized. They are key in informing policy by supporting holistic, multi-disciplinary and evidence based policy implementation. They foster delineation of locales and sectors requiring resources and interventions. About $90 \%$ of the population in Malawi and most of sub-Saharan Africa rely on rain fed agriculture. Due to this overreliance, people's livelihoods and economy are vulnerable to hydrological hazards such as flood and droughts, which account for $70 \%$ of all the hazards in the region. This paper employs indicators to couple exposure, susceptibility, capacity measures and economic, social, physical and environmental components to measure multiple hazard vulnerability. It then utilizes the Community Based Disaster Risk Index in the analysis. Results show that Karonga is an area of medium and high multi hazard vulnerability. The lead contributor to this vulnerability is susceptibility, which manifests itself as high and very high, with predominance in the high levels. Exposure manifests on the lower end of vulnerability spectrum. Lack of capacity is predominantly medium. Socio economic and environmental aspects underlie this susceptibility. Reducing vulnerability in these economies will demand radical programs that target infrastructural investment, socioeconomic empowerment, environmental management and strengthening of institutional capacity.
\end{abstract}

Key-words: Multi hazard vulnerability, Exposure, Susceptibility, Capacity measures, Malawi, Sub Saharan Africa.

\section{INTRODUCTION}

Scientific predictions and evidence affirms that global climate change is likely to increase further the exposure to multiple-risks affecting the magnitude, frequency and spatial distribution of hazardous and disastrous events (IPCC, 2014). Population growth, urbanization and the inability of poor populations to escape from vicious cycle of poverty makes it all more likely there will be an increase in the number of people who are vulnerable to natural hazards with a resulting increase of disasters and environmental emergencies (Guha-Sapir et al., 2004). In the sub-Saharan Africa (SSA), hydro-meteorological hazards tend to be the most dominant.

\footnotetext{
${ }^{1}$ Malawi University of Science and Technology, Ndata School of Climate and Earth Sciences, P.O Box 5196, Limbe, Malawi tamarakamanga@gmail.com

${ }^{2}$ Naresuan University, Centre of Excellence on Energy Technology and Environment, Faculty of Engineering, Tapho Sub-District, Mueang District, Phitsanulok, 65000, Thailand, sarintipt@nu.ac.th

${ }^{3}$ University of Malawi, The Polytechnic, Department of Civil Engineering, Blantyre, Malawi, fmwale@poly.ac.mw

${ }^{4}$ Naresuan University, Faculty of Engineering, Tapho Sub-District, Mueang District, Phitsanulok, 65000,Thailand, tamarakamanga@gmail.com,panub@nu.ac.th
} 
Correspondingly, economies are agro-based with agriculture being primarily rain fed and supporting over $90 \%$ of the farmers (Tchale, 2009). This interplay renders people very vulnerable to any climatic shocks.

Multi hazard vulnerability assessments are encouraged in key government and intergovernmental initiatives and agencies. The Sendai Framework for Disaster Risk Reduction, states that disaster risk reduction needs to be multi hazard, and effective risk reduction is possible if all relevant stresses are taken into consideration and analyzed (UNISDR, 2015). Among many advantages, multi hazard vulnerability offers the function of highlighting locations and sectors requiring more targeted interventions and are a prerequisite for understanding risk and the development of risk reduction and adaptation strategies to extreme events.

Researchers have utilized a wide range of approaches to measure vulnerability over the years. Several European Projects studied hazard and risk mapping techniques for different natural hazards like floods, earthquakes, landslides, forest fires, volcanoes and meteorological extreme events, climate change, etc. (Boukalova, 2005; Klemesova et al., 2014; Pashova et al., 2016). The focus of most past researches had been mainly on single hazards, ignoring the multi hazard measurement. The danger of measuring single hazards over multiple hazards is that they can mislead management priorities, increase vulnerability to other spatially relevant hazards or underestimate vulnerability (Budimir et al., 2014; Gill \& Malamud, 2016; Kappes et al., 2010). Areas under study are Wasambo, Lupembe, Kilipula, Mwakaboko, Mwirang'ombe and Karonga Town communities in Karonga district, northern Malawi.

\section{MATERIALS AND METHODOLOGY}

\subsection{Study Area and Data}

The study focused on communities in Karonga district in Northern Malawi. (Fig. 1). The district hosts a total land area of $3,416 \mathrm{~km}^{2}$, and a population of 365,028 people (National Statistical Office, 2018). Poverty levels according to the fourth Integrated Household Survey (IHS4), collected in 2016-2017, stand at 57.1\%, higher compared to the national average at $51.5 \%$. The main livelihoods are crop farming, livestock production and fishing (Mapoma et al., 2017). Karonga is prone to flooding with the Songwe, North Rukuru, Kibwe, Kasisi, Nyungwe, Wayi, Lufirya and Kyungu being the rivers that commonly floods (UNECA, 2015). Drought is an annual occurrence in the district, with serious impact on food security as $75 \%$ of the households are engaged in crop and animal husbandry (Manda, 2014).

The scale of analysis in this study follows the institutional framework for disaster management in Malawi. Karonga district has one District Civil Protection Committee (DCPC), 6 Area Civil Protection Committee (ACPC) and 46 Village Civil Protection Committee (VCPC) s. The ACPC level was chosen as a scale as of analysis for this study, and all six communes taken into consideration. These are Kilipula, Mwakaboko, Kilipula, Mwirang'ombe, Wansambo and Karonga Town communes.

\subsection{Vulnerability framework}

Vulnerability is multifaceted and definition use commonly depends on the audience and decisions in question. The IPCC defines vulnerability as the propensity or predisposition to be adversely affected (IPCC, 2014). 
According to this definition, vulnerability encompasses three components of exposure, sensitivity and adaptive capacity (IPCC, 2014). UNSIDR (2017) classifies vulnerability into four main types namely: economic, social, physical and environmental. This classification indicates that each social entity has different types of vulnerability, and it is not only the result of the human actions, decisions and choices, but it is the result of the interaction of the different contexts where people live (Alcantara-Ayala, 2002).

In this study, exposure is the presence of people, livelihoods, species or ecosystems, environmental functions, services, and resources, infrastructure, or economic, social, or cultural assets in places and settings that could be adversely affected (IPCC, 2014). While susceptibility is the degree, to which a system is open, liable, or sensitive to a hazard. Capacity Measures /Resilience is the combination of all the strengths, attributes and resources available within an organization, community or society to manage and reduce disaster risks and strengthen resilience (UNSIDR, 2009).

This study assess vulnerability by coupling indicators of susceptibility, exposure, and capacity measures based on IPCC framework of which parameters in each group can be categorized to social, economic, environment and physical discourse following the sustainable development framework. Fig. 1 shows the study process.

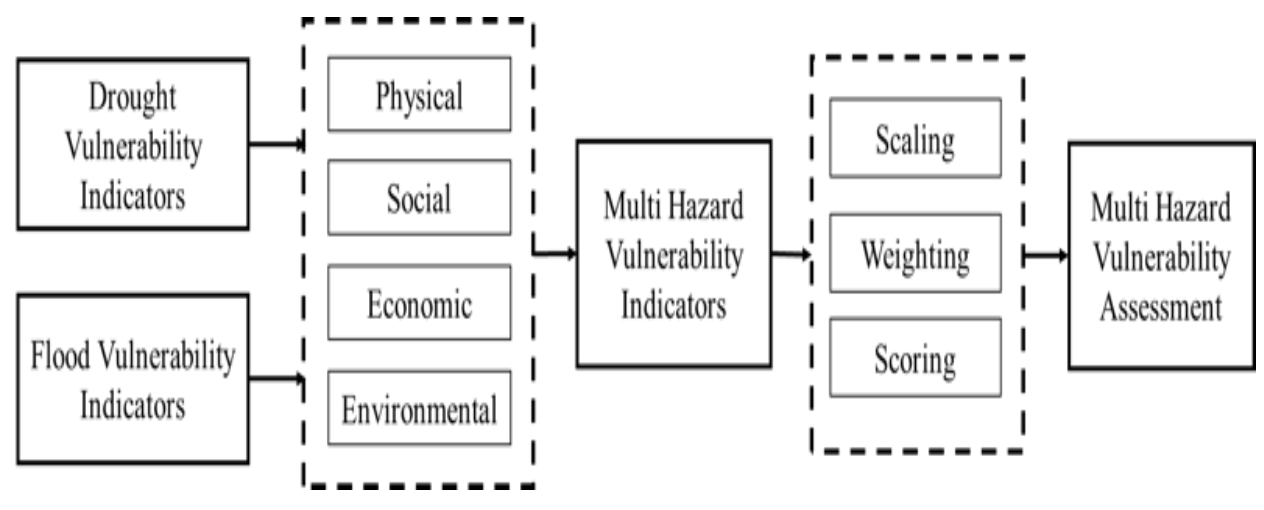

Fig. 1. Study process.

\subsection{Indicators for vulnerability quantification}

Vulnerability is a product of myriad factors (Suwarno et.al., 2019; Fujiki \& Renard, 2018). Factors that are eventually selected in measuring vulnerability are dependent on several factors such as the scale of analysis the aims of the study, relative ease of measurement, validity of the indicator, data availability and methodological approach in building the index (Mwale et al., 2015).

To assess multi hazard vulnerability in this study, flood vulnerability indicators adopted from the CBDRI were coupled with drought indicators drawn from various literature in Sub-Saharan Africa (SSA) (Ahmadalipour \& Moradkhani, 2018; Ahmadalipour et al., 2019; Hahn et al., 2009; Hannaford, 2018; Malcomb et al., 2014; Muyambo et al., 2017; Naumann et al., 2014). Table 1 presents the indicators used. 


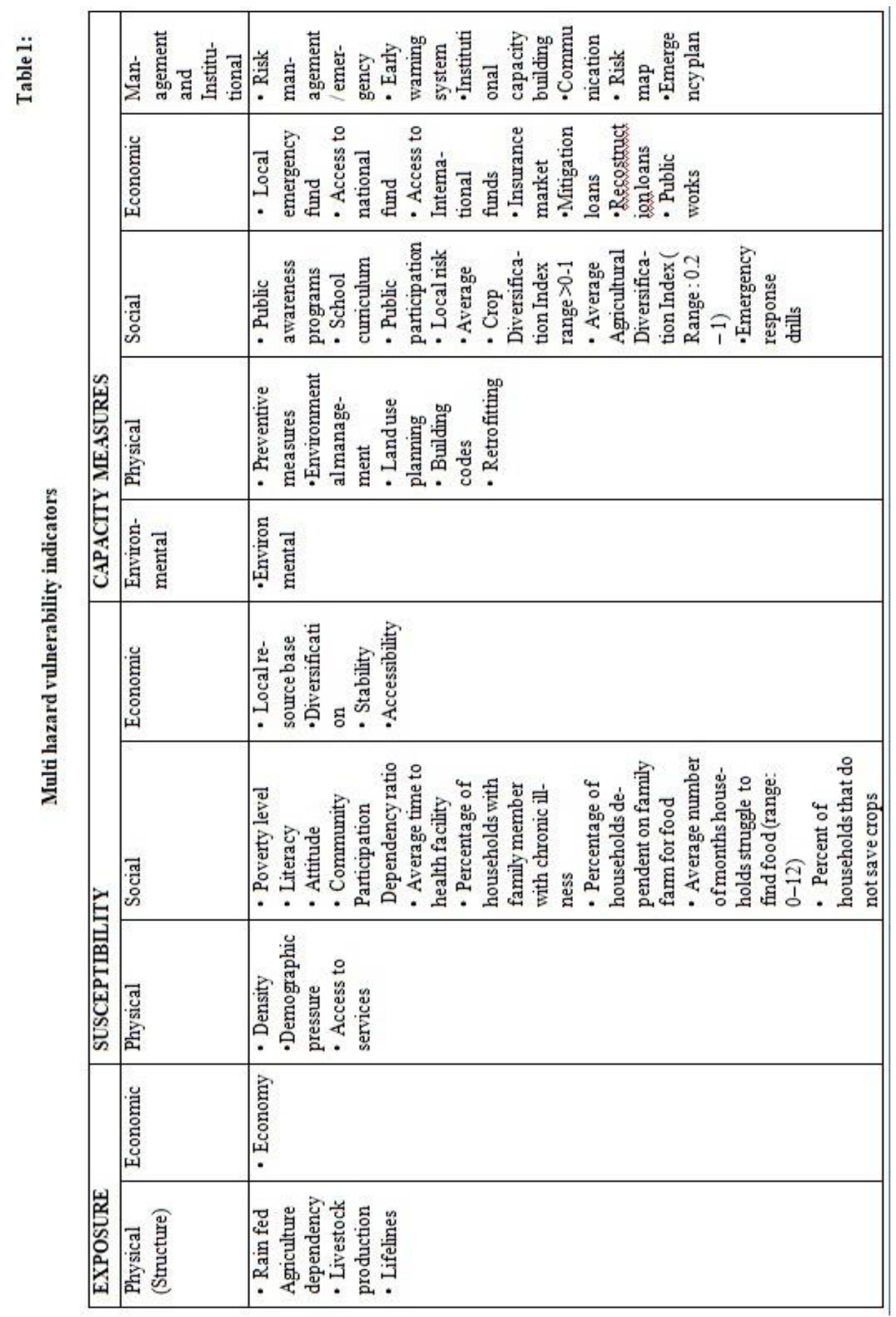




\subsection{Index selection}

This study adapts the Community Based Disaster Risk Index (CBDRI) by Bollin et al (2013). The CBDR was chosen as is applicable in data scarce areas where data for conventional vulnerability assessment is limited. The CBDRI utilizes equations (1) and (2) in its operations.

$$
\begin{aligned}
& \text { CBDRI }=v(H+E+V-C) \\
& H=\sum_{i=1}^{h}\left(w_{i} x_{i}\right), \mathrm{E}=\sum_{j=1}^{q}\left(w_{j} x_{j}\right), S=\sum_{k=1}^{r}\left(w_{k} x_{k}\right), \mathrm{C}=\sum_{y=1}^{z}\left(w_{y} x_{y}\right)
\end{aligned}
$$

Where $\mathrm{H}, \mathrm{E}, \mathrm{V}$ and $\mathrm{C}$ are the hazard, exposure, vulnerability and capacities (resilience) sub components with a range from zero to $100 ; \mathrm{v}=0.33$.

The $\mathrm{v}$ factor keeps the final value within zero and 100. $w$ is a weight reflecting indicator importance in the sub-component. $h q r z$, are the total number of indicators in the hazard, exposure, vulnerability and capacity components respectively. $x$ is a score allocated to the indicator in the sub-component and in the original state (equation 1) is equal to either 1 (low), 2 (medium) and 3 (high). In the adapted form used in this study, the CBDRI uses susceptibility $\mathrm{S}$ in place of vulnerability $\mathrm{V}$ in equation (1). This is because in vulnerability literature, exposure, susceptibility and capacities underlie vulnerability (Adger, 2003; Birkmann, 2013; Fussel \& Klein, 2006; Smit et al., 2000). In addition, since the CBDRI, measures risk directly but as per equation (1), it is possible to disaggregate it to measure vulnerability directly as in equation (3). Thus.

$$
\mathrm{V}=1 / 3[\mathrm{E}+\mathrm{S}+(1-\mathrm{C})]
$$

Where $(1-C)$ is the lack of capacity. For example, if exposure is 0.65 , susceptibility is 0.80 , and lack of capacity is 0.4 , then: $V=(0.60+0.80+0.4) / 3=0.6$. Further, the scores in this study were stretched from 1 - 3 to 1 - 5 as follows: 1 (very low), 2 (low), 3 (medium), 4(high) and 5 (very high). In the original CBDRI, the total sum of weights per sub-component is 33 .

With a maximum score of 3 , this ensures that the subcomponent value does not exceed 100. In this study, with a maximum score of 5, the sum of weights per subcomponent was therefore 20. Since the weights ranged from 1 (less important) to 10 (most important) in which case their sum would exceed 20 , an adjusted weight for the indicator was used. A simple mathematical proportion was the applied. For example, assuming the total number of weights in the susceptibility subcomponent is 50 , and the variable 'poverty level' has a weight of 5 , the adjusted weight for this indicator was $5 / 50 * 20=2$. To dimension single hazard vulnerability by the social, economic, environment and physical dimensions $\left(\mathrm{V}_{\mathrm{j}}\right)$, variables were rearranged and then measured by equation (4) and (5)

$$
\mathrm{V}_{\mathrm{j}}=\left[\left(\mathrm{V}_{\mathrm{Ej}}+\mathrm{V}_{\mathrm{Sj}}+\left(1-\mathrm{V}_{\mathrm{Cj}}\right)\right)\right] / 3
$$

Where, $\mathrm{V}_{\mathrm{Ej}}=\sum_{i=1}^{m}\left[x_{i}\left(20 \frac{w i}{\sum_{i=1}^{m} w i}\right)\right], \mathrm{V}_{\mathrm{Sj}}=\sum_{i=1}^{n}\left[x_{1}\left(20 \frac{w i}{\sum_{i=1}^{m} w i}\right)\right]$,

$$
\mathrm{V}_{\mathrm{Cj}}=\sum_{i=1}^{i=1}\left[x_{i}\left(20 \frac{w i}{\sum_{i=1}^{m} w i}\right)\right]
$$

Where $V_{E J}, V_{S j}$ and $V_{C j}$ are the vulnerability due to exposure $\left(E_{j}\right)$, susceptibility $\left(S_{j}\right)$, and capacities $\left(\mathrm{C}_{\mathrm{j}}\right)$, within the social, economic, environmental and physical sub-component $\mathrm{V}_{\mathrm{j}}$. $\mathrm{X}_{\mathrm{i}}$ is the score and 
$\mathrm{W}_{\mathrm{i}}$ is the weight. $\mathrm{m}, \mathrm{n}$ and $\mathrm{p}$ are the number of variables in the exposure $(\mathrm{Ej})$, susceptibility $(\mathrm{Sj})$ and capacities $(\mathrm{Cj})$ respectively of the sub-component $\mathrm{V}_{\mathrm{j}}$. $\mathrm{V}_{\mathrm{EJ}}, \mathrm{V}_{\mathrm{Sj}} \mathrm{V}_{\mathrm{Cj}}$ and $\mathrm{V}_{\mathrm{j}}$ range from 0 to 100 (Mwale et al., 2015).

For the ultimate multi hazard vulnerability measurement, this study coupled variables from the two hazards that is flood and drought, then dimensioned them into susceptibility, exposure and lack of capacity and further into economic, physical, social and environmental aspects as explained in equations 1 - 5. Vulnerability levels obtained were scaled based on quantiles. Thus $0-0.2$ represented very low vulnerability, $>0.2-0.4$ represented low vulnerability, $>0.4-0.6$ equated medium vulnerability, while $>0.60-0.80$ and $>0.80-1$ represented high and very high vulnerability respectively.

\subsection{Data Collection}

For each community, primary data was sourced through a structured questionnaire administered to a group of experts and knowledgeable people representing the community as recommended by Bollin et al., (2003). The knowledgeable people were mainly from ADC. ADC members are people with various qualifications mainly from police, Community Based Organizations (CBOs) and Government departments. This study also had local government representatives, chiefs and some ordinarily community members. For each commune, one questionnaire was administered, and members agreed on the weight and score. Nine people were interviewed in each of the six communes under study, which is about $70 \%$ of ADC composition.

Secondary data such as dependency ratio, population density, population growth rate, access to water services, and literacy levels were obtained from the fourth Integrated Household Surveys and the 2008 and 2018 population and housing census data. Agricultural and Crop diversification index were calculated using data obtained from the Ministry of Agriculture, Irrigation and Water Development. The percentage of forested area as well as land under cultivation for a community was derived with Geographic Information System (GIS), from land cover data sourced at the Regional Centre for Mapping of Resources for Development (RCMRD) geoportal.

\section{RESULTS AND DISCUSSIONS}

\subsection{Results}

\subsubsection{Exposure}

Exposure to multi hazards is generally low in Karonga, manifesting in the very low (0$0.2)$, low (>0.2-0.4) and medium (>0.4-0.6) levels, but with a predominance in the low level. Magnitudes for this vulnerability ranged $0.2-0.53$ across communes. The low level of exposure in the district in general may arise from Karonga being predominantly rural as most districts in Malawi. Karonga Town is the commune with highest exposure, (Fig. 2). This can be explained from the commune being a town and thus more items in harm's way.

\subsubsection{Susceptibility}

The high level of vulnerability in Karonga is principally attributable to high levels of susceptibility (Fig. 2). Susceptibility magnitudes for multi hazard vulnerability ranged 0.79 -0.84 , manifesting as high $(>0.6-0.8)$, and very high $(>0.80-01)$, but with a predominance in the very high level (Fig. 2). 


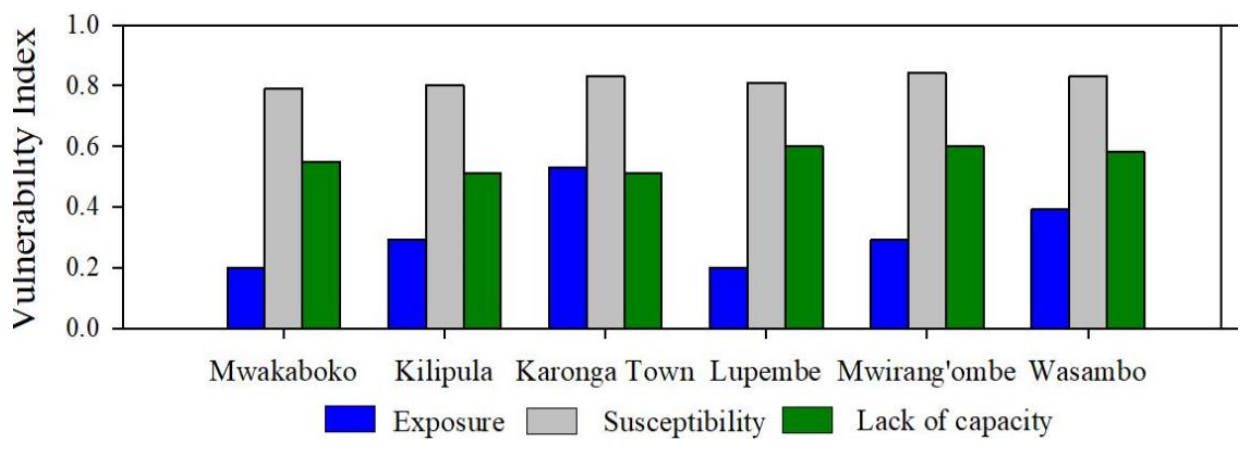

Fig. 2. Multi hazard vulnerability magnitudes.

\subsubsection{Lack of Capacity}

Resilience is generally medium in Karonga communes. Magnitudes ranges $0.4-0.49$. Lupembe, Mwirang'ombe and Wasambo communes have slightly lower resilience as compared to Karonga Town, Kilipula and Mwakaboko (Fig. 2). The slightly higher resilience in Kilipula, Mwakaboko and Karonga Town may be attributable to the fact that these communes are considered as high flood prone areas hence, they are more targeted for programs involving disaster risk management, consequently increasing their capacity measures / resilience.

\subsubsection{Total Vulnerability}

Karonga depicts a medium $(>0.4-0.6)$ to high $(>0.6-0.8)$ level of vulnerability to multi hazards, with a predominance in the medium range (Fig. 3). The magnitudes for this vulnerability ranged $0.51-0.62$.

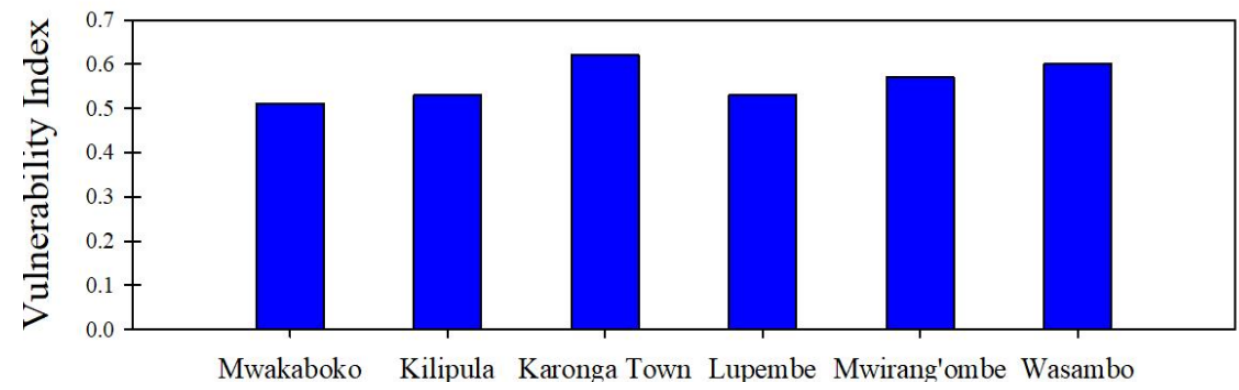

Fig. 3. Total multi hazard vulnerability

\subsection{Discussion}

As seen from the results, vulnerability to multi hazards in Karonga is quite significant, manifesting in the medium to high range. This high vulnerability emanates from high susceptibility levels underlined by socio economic and environmental factors, mainly originating from low levels of income. These findings are in line with vulnerability literature to climatic shocks in developing countries.

For instance, Yiran et al. (2017), mapped the vulnerability to multiple hazards in the Savannah Ecosystem in Ghana, and their study established that the high vulnerabilities in the area were because of high susceptibilities and low adaptive capacities. Similar to the current study, the underlying causes of vulnerability in the Savanna Ecosystem in Ghana 
were socio economic factors. Zacarias (2019), in his quest to understand community vulnerability to climate change and variability at a coastal municipality in southern Mozambique found out that vulnerability is susceptibility driven and is especially derived from susceptibilities of physical, financial and social capitals. Comparably, Ahmadalipour et al. (2019) found out that vulnerability to drought is specifically high in the Sub Saharan countries than the Sahel region despite the latter being an area of high water stress. The underlying causes of this vulnerability were generally socio economic factors, as well as limitations in energy and infrastructure development in the region. Similarly, Mohmmed et al. (2018) substantiated that Darfur region is highly exposed and sensitive to drought risks. The high vulnerability was mainly due to susceptibility factors such as less crop diversity, higher land degradation, frequent droughts, and high poverty levels.

In contrast to susceptibility, exposure is much lower falling in the very low to medium levels but with predominance of low levels. This may be unexpected. However, the low level of exposure in comparison may be explained from the low value of assets in harm's way. It is to be noted that Karonga, as most of the country and a larger part of SSA, is predominantly rural. This is in agreement with figures on economic loses on hazards in developing countries in comparison to developed countries. For instance, CRED 2018's report on economic losses by disasters from 1998 to 2017 indicates that only US\$ 21 billion were lost due to climate related disaster in the period 1998 to 2017, in comparison to US\$1432 billion lost from developed countries during the same time (Wallemacq et al., 2018).

The lack of capacity was expected to be very high, but contrary to expectations, capacities are predominantly medium. It is to be noted though that except for public participation and presence of decentralized institutions, many attributes from physical, economic and to a large extent institutional aptitude are very limited. The underlying factors are limited alternative agricultural technologies, nonexistence of risk loans and other microfinances for disaster management in the communes as well as low physical capacities such as retrofitting, building codes, and other preventive measures. Thus, effectively, capacities to manage disasters in developing countries remain in a precarious state.

The Sustainable Development Framework is equally supportive of the findings. There are heightened levels in environmental, social as well as economic vulnerabilities, underscoring the socio-economic and environmental dimension of vulnerabilities in rural communities in developing countries. Socio economic aspects like poverty, makes people have limited or no access to financial support that is essential to protect them and their assets from hazards, and disaster insurance are not a priority for them, hence heightened susceptibilities. Several studies in SSA have examined income as a determinant of vulnerability using various proxies, including Gross Domestic Product (GDP), Gini index, and household income and have found high poverty levels to be the underlying causes of vulnerability in the region (Adger, 2003; Malcomb et al., 2014; Mohmmed et al., 2018; Mwale et al., 2015; Vincent, 2004).

Higher susceptibility in the environmental component, agrees with the current state of affairs for the environment in Sub-Saharan Africa. For instance, Keenan et al. (2015), documented that the highest net losses in forest area between 2010 and 2015 in Africa were in Nigeria (410 K ha y1), Tanzania (372 K ha y1), Zimbabwe (312 K ha y1) and Democratic Republic of Congo (311 K ha y1). Generally, the enterprises culprit for this are agriculture land for farmland, charcoal burning, brick making, and other small-scale businesses that puts a lot of pressure on the environment (DeFries et al., 2010; Fisher, 2010; Mwale et al., 2015). 
Vulnerability literature suggests that exposure to multi-hazards or repeated exposure to single hazards modifies vulnerability magnitudes. In China for example, Tian et al. (2019) examined temporal trends in resilience to landslides, rock falls and debris flows over a 15 year period. He found that not only did community resilience generally decrease over the years, it increased in periods following catastrophic hazards and it was correlated to original adaptive capacity of the communities, their coping and adaptive capacities related to infrastructure investment, availability of television services, labor employment, medical condition and Engel coefficient.

While floods and droughts may not coincide in time, they often coincide spatially and therefore may have the compounding effect on vulnerability. However, as demonstrated in this study, multi vulnerability magnitudes fall in relatively the same ranges with single hazard vulnerability (Mwale et al., 2015).

In developing countries where such factors as infrastructural investment, poverty, employment and environmental factors are structural deficiencies, it is unlikely vulnerability may vary significantly within the span of the sequential occurrence of floods and droughts, supporting the findings herein.

\section{CONCLUSIONS}

Vulnerability to multi-hazards of floods and droughts in Karonga, Malawi is medium to high. It is susceptibility driven rooted in social, economic and largely, environmental factors. Measurement of vulnerability to single natural hazards including floods and droughts has been conducted in Sub-Saharan Africa (Ahmadalipour \& Moradkhani, 2018; Mwale et al., 2015; Williams et al., 2018; Zacarias, 2019). More importantly, a coupled IPCC and UNISDR framework has been applied tested on floods (Mwale et al. 2015). The patterns depicted from these studies are in general agreement with a multi-hazard spectrum found herein, both in patterns and in magnitudes. This underscores the dominance of structural factors to vulnerability of people in developing countries irrespective of the type of hazard exposed to. Ultimately, radical programs that target infrastructural investments, socio-economic empowerment, and environmental protection and institutional capacity can play a vital role in reducing vulnerability to these climatic hazards.

The multi hazard assessment applied in this study is recommended to areas facing multiple hazards, such as the Yom river basin in Thailand. This approach is also highly applicable to areas with limited data and resources like most of the developing countries.

\section{ACKNOWLEDGEMENT}

This study was partly supported by Advancing Co-design of Integrated Strategies with Adaptation to Climate Change in Thailand (ADAP-T), Grant Number: JPMJSA1502 supported by the Science and Technology Research Partnership for Sustainable Development (SATREPS), JST-JICA.

\section{REFERENCES}

Adger, W. N. (2003). Social aspects of adaptive capacity Climate change, adaptive capacity and development (pp. 29-49): World Scientific.

Ahmadalipour, A., \& Moradkhani, H. (2018). Multi-dimensional assessment of drought vulnerability in Africa: 1960-2100. Science of The Total Environment, 644, 520-535. doi: https://doi.org/10.1016/j.scitotenv.2018.07.023 
Ahmadalipour, A., Moradkhani, H., Castelletti, A., \& Magliocca, N. (2019). Future drought risk in Africa: Integrating vulnerability, climate change, and population growth. Science of The Total Environment, 662, 672-686. doi: 10.1016/j.scitotenv.2019.01.278

Alcantara-Ayala, I. (2002). Geomorphology, natural hazards, vulnerability and prevention of natural disasters in developing countries. Geomorphology, 47(2-4), 107-124.

Birkmann, J., Cardona, O. D, Carreño, Martha Liliana, Barbat, Alex H, Pelling, Mark, Schneiderbauer, Stefan, Kienberger, StefanKeiler, Margareth, Alexander, David E, Zeil, Peter, . (2013). Framing vulnerability, risk and societal responses: the MOVE framework. Natural Hazards, 67(2), 193-211.

Boukalova, Z. (2005) Applied multi Risk Mapping of Natural Hazards for Impact Assessment, ARMONIA Project.

Budimir, M., Atkinson, P., \& Lewis, H. (2014). Earthquake-and-landslide events are associated with more fatalities than earthquakes alone. Natural Hazards, 72(2), 895-914.

DeFries, R. S., Rudel, T., Uriarte, M., \& Hansen, M. (2010). Deforestation driven by urban population growth and agricultural trade in the twenty-first century. Nature Geoscience, 3(3), 178.

Fisher, B. (2010). African exception to drivers of deforestation. Nature Geoscience, 3(6), 375.

Fujiki, K., \& Renard, F. (2018). A Geographic Analysis of Post-Disaster Social Impacts on Municipal Scale - A Case study of potential Major Flood in the Paris Region (France). Geographia Technica, 13(2), 31-51.

Fussel, H. M., \& Klein, R. J. T. (2006). Climate change vulnerability assessments: An evolution of conceptual thinking. Climatic Change, 75(3), 301-329. doi: 10.1007/s10584-006-0329-3

Gill, J. C., \& Malamud, B. D. (2016). Hazard interactions and interaction networks (cascades) within multi-hazard methodologies. Earth System Dynamics, 7(3), 659-679.

Guha-Sapir, D., Hargitt, D., \& Hoyois, P. (2004). Thirty years of natural disasters 1974-2003: The numbers: Presses univ. de Louvain.

Hahn, M. B., Riederer, A. M., \& Foster, S. O. (2009). The Livelihood Vulnerability Index: A pragmatic approach to assessing risks from climate variability and change-A case study in Mozambique. Global environmental change, 19(1), 74-88.

Hannaford, M. J. (2018). Long-term drivers of vulnerability and resilience to drought in the ZambeziSave area of southern Africa, 1505-1830. Global and planetary change, 166, 94-106. doi: https://doi.org/10.1016/j.gloplacha.2018.05.001

IPCC. (2014). ANNEX II. In K. J. Mach, Planton S. Von Stechow C (Ed.).

Kappes, M., Keiler, M., \& Glade, T. (2010). From single-to multi-hazard risk analyses: a concept addressing emerging challenges.

Keenan, R., Reams, G., Frédéric, A., Freitas, J., Grainger, A., \& Lindquist, E. (2015). Dynamics of global forest area: Results from the FAO Global Forest Resources Assessment 2015. Rodney J. Keenan,Gregory A. Reams, Frédéric Achard, Joberto V. de Freitas, Alan Grainger, Erik Lindquist, 352.

Klemesova, K., Kolar, M., \& Andrask, I. (2014). Using GIS in the flood management - flood map. Geographia Technica, 9(2), 44-53.

Malcomb, D. W., Weaver, E. A., \& Krakowka, A. R. (2014). Vulnerability modeling for sub-Saharan Africa: An operationalized approach in Malawi. Applied Geography, 48, 17-30. doi: https://doi.org/10.1016/j.apgeog.2014.01.004

Manda, M. Z. (2014). Where there is no local government: addressing disaster risk reduction in a small town in Malawi. Environment and Urbanization, 26(2), 586-599.

Mohmmed, A., Zhang, K., Kabenge, M., Keesstra, S., Cerdà, A., Reuben, M., . . . Ali, A. A. (2018). Analysis of drought and vulnerability in the North Darfur region of Sudan. Land Degradation \& Development, 29(12), 4424-4438.

Muyambo, F., Jordaan, A. J., \& Bahta, Y. T. (2017). Assessing social vulnerability to drought in South Africa: Policy implication for drought risk reduction. Jàmbá: Journal of Disaster Risk Studies, 9(1), 1-7.

Mwale, F. D., Adeloye, A. J., \& Beevers, L. (2015). Quantifying vulnerability of rural communities to flooding in SSA: A contemporary disaster management perspective applied to the Lower Shire 
Valley, Malawi. International Journal of Disaster Risk Reduction, 12, 172-187. doi: https://doi.org/10.1016/j.ijdrr.2015.01.003

Naumann, G., Barbosa, P., Garrote, L., Iglesias, A., \& Vogt, J. (2014). Exploring drought vulnerability in Africa: an indicator based analysis to be used in early warning systems. Hydrol. Earth Syst. Sci., 18(5), 1591-1604. doi: 10.5194/hess-18-1591-2014

Pashova, L., Kouteva-Guentcheva, M., \& Bandrova, T. (2016). Towards Maping Multi-Hazard Vulnerability of Natural Disasters for the Bulgarian Territory Paper presented at the 6th International Conference on Cartography and GIS.

Smit, B., Burton, I., Klein, R. J., \& Wandel, J. (2000). An anatomy of adaptation to climate change and variability Societal adaptation to climate variability and change (pp. 223-251): Springer.

Suwarno, Sutomo \& Aditama, M.R. (2019). The analysis of the Landslide Vulnerability Sub Watersheds Arus in Banyumas Regency Geographia Technica, 14(2), 112-119.

Tchale, H. (2009). The efficiency of smallholder agriculture in Malawi. AFJARE, 3(2), 101-121.

Tian, C.-s., Fang, Y.-p., Yang, L. E., \& Zhang, C.-j. (2019). Spatial-temporal analysis of community resilience to multi-hazards in the Anning River basin, Southwest China. International Journal of Disaster Risk Reduction, 101144.

UNECA. (2015). Assesment Reort on Mainstreaming and implementing disaster risk reduction measures in Malawi Addis Ababa, Ethiopia: United Nations Economic Commmision for Africa.

UNSIDR. (2009). Terminology on disaster risk reduction from united nations international strategy for disaster reduction.

UNSIDR. (2017). Terminology on disaster risk reduction from United Nations International Strategy for Disaster Reduction.

Vincent, K. (2004). Creating an index of social vulnerability to climate change for Africa. Tyndall Center for Climate Change Research. Working Paper, 56(41).

Wallemacq, P., Below, R., \& McLean, D. (2018). UNISDR and CRED report : Economic Losses , Poverty \& Disasters (1998 - 2017).

Williams, P. A., Crespo, O., \& Abu, M. (2018). Assessing vulnerability of horticultural smallholders' to climate variability in Ghana: applying the livelihood vulnerability approach. Environment, Development and Sustainability, 1-22.

Yiran, G. A., Stringer, L. C., Attua, E. M., Evans, A. J., Challinor, A. J., \& Gyasi, E. A. (2017). Mapping vulnerability to multiple hazards in the savannah Ecosystem in Ghana. Regional environmental change, 17(3), 665-676.

Zacarias, D. A. (2019). Understanding community vulnerability to climate change and variability at a coastal municipality in southern Mozambique. International Journal of Climate Change Strategies and Management, 11(1), 154-176. 